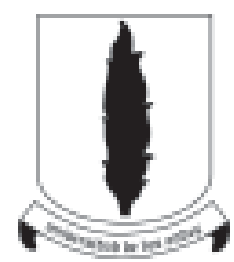

FACULTAD DE INGENIERÍA

\section{REVISTA DE INGENIERIA}

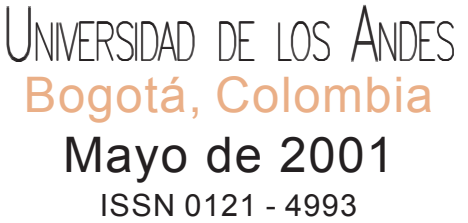

Número 13
José Tiberio Hernández Peñalosa

Decano

Rafael Gómez

Vicedecano de Pregrado

Gonzalo Torres

Decano Asociado de Investigación y Consultoría

Alain Gauthier

Director de Posgrados

Claudia Cárdenas Gutiérrez

Secretaria General

Comité Editorial

Olga Lucía Giraldo, José María Escobar, Mauricio Duque, Eugenio Giraldo, Juan Saldarriaga, José Ignacio Huertas, Jaime Loboguerrero, Arcesio Lizcano

Editor

Alberto Sarria

Diseño e Impresión

Siglo XXI Impresores Ltda.

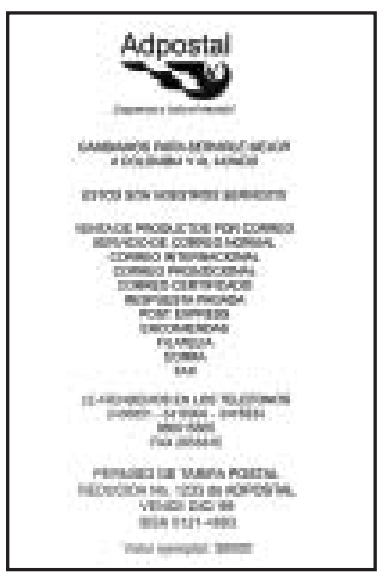

PERMISO DE TARIFA POSTAL REDUCIDA NO. 1233 DE ADPOSTAL

VENCE DIC/2001

ISSN 0121-4993

Valor ejemplar: $\$ 20.000$

\section{ÍNDICE GENERAL}

\section{Sección de IngenIería}

Asignacion de rutas de vehículos para un sistema de recoleccion de residuos solidos en la acera 5

Modelación de balances hídricos de ciénagas fluviales y costeras colombianas. I: Fundamentos técnicos

La acumulación de basuras como material geotécnico: I: fundamentos técnicos

Revisión de los criterios de confiabilidad del código de diseño estructural como alternativa para la competitividad

Emisión de compuestos orgánicos volátiles (VOC) durante el compostaje de biosólidos provenientes de plantas de tratamiento de aguas residuales domésticas 37

Diseño sísmico por desempeño en Colombia 45

Incidencia de los sistemas de control en la captación de capital humano 52

Situación energética general del sector de los plásticos en Bogotá 58

Mejoramiento de la red de transmisión nacional con la utilización de nuevas tecnologías 66

Vulnerabilidad sísmica de puentes en

Colombia y estrategias para rehabilitacion 74

Tesis de magíster segundo semestre de 2000 81

Resultados convocatoria nacional para el escalafonamiento de grupos y centros de investigación científica y tecnológica 2000

VI Congreso Nacional de

Estudiantes de Ingeniería Mecánica 


\begin{tabular}{|c|c|}
\hline \multicolumn{2}{|c|}{$\begin{array}{l}\text { FACULTAD DE INGENIERÍA } \\
\text { UNIVERSIDAD DE LOS ANDES }\end{array}$} \\
\hline \multirow[t]{6}{*}{ Programas de Pregrado: } & Ingeniería Civil y Ambiental \\
\hline & Ingeniería Eléctrica y Electrónica \\
\hline & Ingeniería Industrial \\
\hline & Ingeniería Mecánica \\
\hline & Ingeniería Química \\
\hline & Ingeniería de Sistemas y Computación \\
\hline Programas de Magíster en Ingenierías: & $\begin{array}{l}\text { Civil, Eléctrica y Electrónica, Electrónica y de } \\
\text { Computadores, Industrial, Mecánica, Sistemas y } \\
\text { Computación }\end{array}$ \\
\hline \multicolumn{2}{|l|}{ Programa de Doctorado en Ingeniería } \\
\hline \multicolumn{2}{|c|}{$\begin{array}{c}\text { Decanatura de Ingeniería } 33243 \text { 27/28/29/31 Fax } 3324230 \\
\text { e-mail: reingeri@uniandes.edu.co }\end{array}$} \\
\hline Sa1 & de Bogotá \\
\hline
\end{tabular}

\section{LA REVISTA INVITA A SUS LECTORES A COMENTAR EL MATERIAL ESCRITO ENVIANDO CARTAS A REVISTA DE INGENIERÍA -CITEC- \\ Carrera 65B No. 17A - 11 \\ SU COLABORACIÓN CON PAUTA PUBLICITARIA SERÁ MUY BIEN RECIBIDA POR LA REVISTA DE INGENIERÍA.}

LE AGRADECEMOS SU APORTE. 

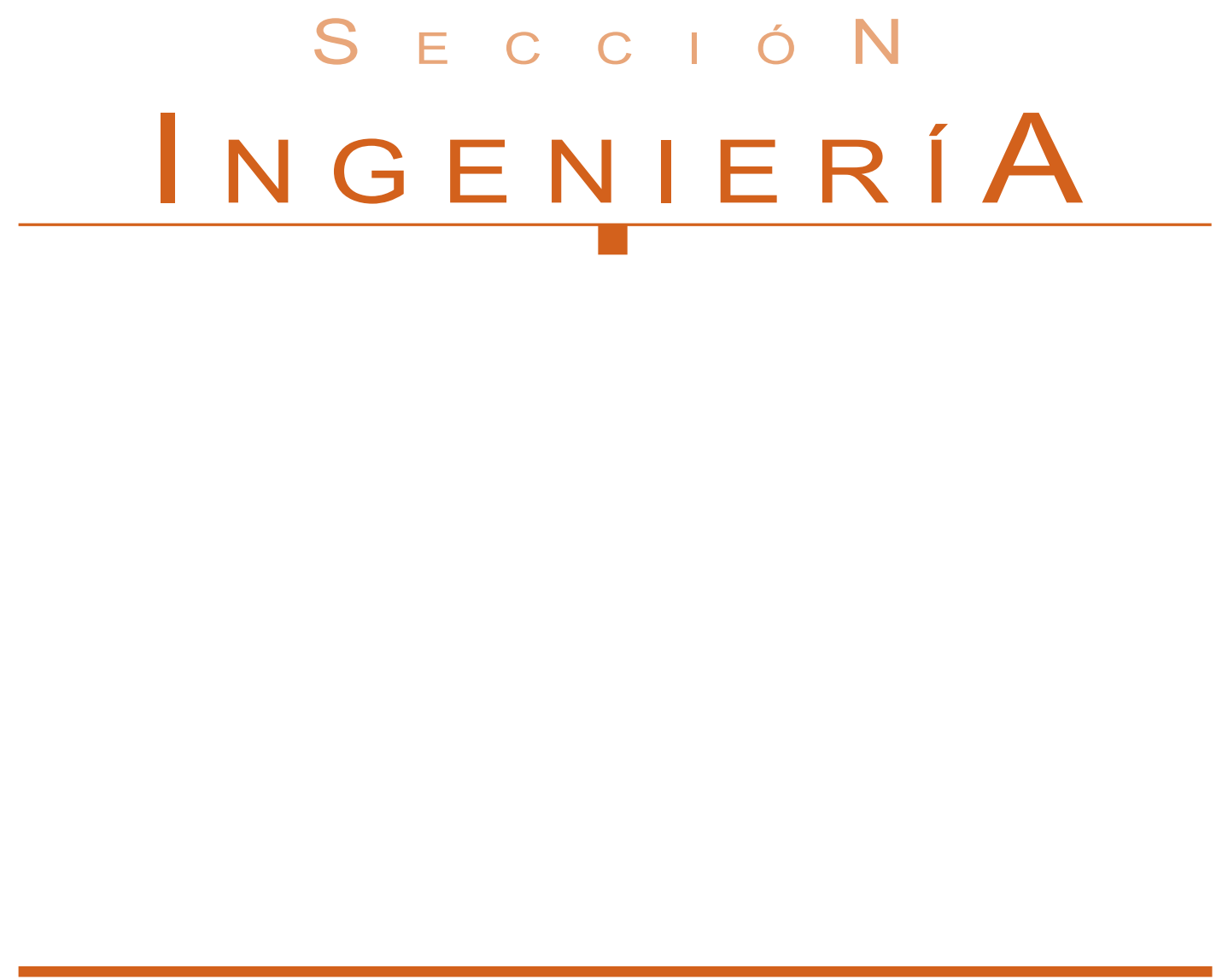


\title{
Asignación de Rutas de Vehículos para un Sistema de Recolección de Residuos SÓLIDOS EN LA ACERA
}

\author{
Alexander Ayala Rodríguez ${ }^{1}$
}

Edgar Gonzáles Butrón²

\begin{abstract}
Solid waste collection problems are of increasing concern to expanding cities and sectors with high volumes of waste generation. This paper deals with the optimization of a collection system in the sidewalk for urban sectors. This is a combinatorial problem in which all arcs of a mixed network are traversed, subject to vehicle capacity and time restrictions. A flexible formulation is proposed which works with separate collection and transportation networks. A sequencing scheme to assign routes is also proposed. The performance of the model is analyzed under different scenarios and applied to a collection zone of Bogotá with satisfactory results.
\end{abstract}

\section{Palabras clave}

Asignación de rutas, recolección de residuos sólidos, tour de Euler, Problema del Cartero Chino, Sistema de Información Geográfica (SIG)

\section{INTRODUCCIÓN}

El crecimiento acelerado de la población en los últimos años, así como el proceso de industrialización han aumentado la generación de residuos, haciendo que la logística de recolección sea más compleja, hoy en día la generación de residuos por persona se estima entre 500 y $1.000 \mathrm{gr} / \mathrm{hab} /$ día, aunque este problema siempre ha existido en la actualidad se ha convertido en un aspecto crítico debido a los altos costos de transporte y mano de obra, en 1992 se estimó que el costo de recolección puede llegar a representar entre un 50 y un $70 \%$ de los costos totales del manejo de residuos sólidos (Tchobanoglous [1]), en Santafé de Bogotá para el año de 1997 este costo fue del $64.3 \%$.

El problema de asignación de rutas de vehículos tienen como objetivo el desarrollar una secuencia de recolección para una flota de vehículos que satisfaga la oferta de los clientes. Este problema es de tipo NP, es decir, que no se puede resolver en tiempo polinomial.

En la formulación de modelos matemáticos para la planeación y asignación de rutas de vehículos de recolección de residuos sólidos se destaca el estudio realizado por Beltrami \& Bodin [2] quienes en 1974 exploraron las técnicas desarrolladas para solucionar este tipo de problemas, motivando el desarrollo de otras investigaciones debidas a Shuster \& Schur [3], Male \& Liebman [4], Bodin \& Golden [5], Eisentein \& Iyer [6] y Or \& Curi [7].

El uso de modelos matemáticos asociados con la capacidad de análisis espacial de los Sistemas de Información Geográfica es el nuevo enfoque de los estudios relacionados con la asignación de rutas de vehículos para la recolección de residuos sólidos, ejemplo de ello son los trabajos realizados por Massie [8], Cargin \& Dwyer [9], Osegueda [10] y Chang [11].

1 Alexander Ayala Rodríguez, MsC. Ingeniería Industrial, Universidad de los Andes.

2 Profesor Asistente, Departamento de Ingeniería Industrial, Universidad de los Andes. 
Para determinar el tipo de algoritmo a ser utilizado en el proceso de asignación de rutas de vehículos es necesario hacer un análisis de la red y de los resultados que se esperan. Si un vehículo es el que debe ser dirigido a través de todos los nodos de la red se tiene un NRP (Node Routing Problem) tal como el TSP (Traveling Salesman Problem), Laporte [12], en cambio sí un vehículo debe recorrer todos los arcos de la red se tiene un ARP (Arc Routing Problem) como el CPP (Chinese Postman Problem) Edmons \& Johnson [13] y Eiselt [14].

\section{Modelo de Asignacion de Rutas de Vehiculos}

\subsection{Generalidades}

El problema de asignación de rutas de vehículos para un sistema de recolección de residuos sólidos en la acera puede considerarse como una variante del CPP, Problema del Cartero Chino, en el cual todos los arcos de una red mixta deben ser recorridos por lo menos una vez. Para ello se requiere que el grafo cumpla con las siguientes condiciones que garanticen la conformación de un Tour de Euler (Evans \& Minieka [15]):

1 El grafo G debe ser conectado, permitiendo el traslado entre cualquier par de nodos.

2 Cada nodo del grafo $\mathrm{G}$ debe ser de grado par, es decir, el número de arcos que inciden en un nodo es igual al número de arcos que salen del mismo.

3 Para cada subconjunto $\mathrm{S}$ de nodos, la diferencia entre el número de arcos dirigidos desde $\mathrm{S}$ a su complemento y el número de arcos dirigidos desde el complemento de $\mathrm{S}$ a $\mathrm{S}$ es menor o igual al número de arcos no dirigidos para $\mathrm{S}$ y su complemento.

En este trabajo se plantea una formulación novedosa para resolver el problema ARV. La solución consiste en agregar una nueva variable de decisión $\mathrm{Y}_{\mathrm{ijvk}}$ que representa una nueva red idéntica a la definida por la variable $\mathrm{X}_{\mathrm{ijvk}}$ (Figura 1), en donde la red formada por los $\mathrm{X}_{\mathrm{ijvk}}$ representa la red de recolección (carga) y la red formada

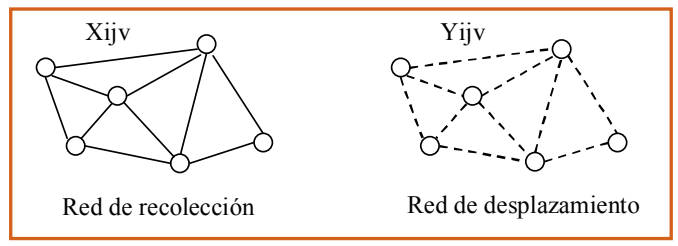

Figura 1. Ilustración de las variables de decisión. por los $\mathrm{Y}_{\mathrm{ijjk}}$ representa la red de desplazamiento o transporte, lo cual permite diferenciar claramente si un vehículo atraviesa un arco realizando actividades de recolección ó si el vehículo utiliza este arco como un punto de tránsito que le permite desplazarse de un nodo a otro.

\subsection{Información de entrada}

- Configuración de la red: Permite convertir el mapa de una región geográfica en una red formada por un conjunto de nodos y arcos para lo cual se requiere conocer el sentido de circulación de las vías, distancia entre puntos de recolección, características del tráfico, estado de las vías, velocidad promedio de circulación, ubicación del garaje o base de operaciones y del sitio de disposición final.

- Estimación de la demanda: La demanda varía en cada punto de recolección por ello para su estimación es necesario conocer el área total (ha), la densidad de población (hab / ha), la tasa de generación de residuos sólidos $(\mathrm{Kg} / \mathrm{hab} *$ día $)$.

- Tiempos de proceso: Tiempos de viaje entre garajemicroruta, microruta-relleno y relleno-garaje, tiempo en microruta (que puede ser de recolección ó desplazamiento) y tiempo de descarga ó de permanencia en el relleno sanitario.

- Disponibilidad del vehículo: Determinada por el tamaño de la flota de vehículos (número de vehículos), la capacidad de cada tipo de vehículo (yardas cúbicas, $1 \mathrm{yd}^{3}=0.7645 \mathrm{~m}^{3}$ ) y la densidad de la basura en el vehículo recolector $\left(\mathrm{Kg} / \mathrm{m}^{3}\right)$.

- Disponibilidad de la tripulación: Determinada por la duración de la jornada de trabajo (horas/hombre), el tamaño de la tripulación (hombres / tripulación) y el número de viajes que puede realizar una tripulación en un día.

- Costos: El problema puede involucrar parámetros adicionales tales como costos de operación, mantenimiento, administración, inversión y comercialización. ya sea por tonelada recolectada o por kilómetro recorrido.

\subsection{Formulación matemática}

El modelo de Asignación de rutas de vehículos para un sistema de recolección de residuos sólidos en la acera posee una estructura similar a la presentada por Chang [11], la notación utilizada es la siguiente: 
$X_{i j v k}$ : Variable de decisión entera para la selección del arco $(i, j)$ en la red de recolección correspondiente al k-ésimo recorrido (tour) de un vehículo recolector tipo v.

$Y_{i j v k}$ : Variable de decisión entera para la selección del $\operatorname{arco}(i, j)$ en la red de desplazamiento correspondiente al k-ésimo recorrido (tour) de un vehículo recolector tipo v.

$D_{i j}$ : Distancia a través del arco $(\mathrm{i}, \mathrm{j}),(\mathrm{Km}, \mathrm{mts})$.

$Q_{i j}$ : Cantidad de residuos a ser recolectados a través del arco (i,j), (Ton, $\mathrm{Kg}$ )

$C_{v}$ : Capacidad de un tipo especifico de vehículo recolector (Ton, $\mathrm{Kg}$ ).

$V: \quad$ Número total de tipos de vehículos de una flota.
$K_{v}: \quad$ Numero máximo de servicios que puede realizar un vehículo en un día.

$T_{v k}$ : Máximo límite de tiempo de recolección en el késimo viaje para un vehículo recolector tipo v, (hrs, min).

$t c_{i j}$ : Tiempo de carga ó recolección a lo largo del arco (i, j) para el k-ésimo viaje en un vehículo recolector tipo $\mathrm{v}$, (hrs, $\mathrm{min}$ ).

$t d_{i j}$ : Tiempo de viaje o desplazamiento a lo largo del arco (i, j) para el k-ésimo viaje en un vehículo recolector tipo $\mathrm{v}$, (hrs, $\mathrm{min}$ ).

$N$ : Número total de nodos en la red.

I : Conjunto de todos los nodos alrededor del nodo i en la red.

$N^{\prime}=$ Número total de nodos en el vecindario del nodo i en la red.

$I^{\prime}=$ Conjunto de todos los nodos alrededor del nodo $\mathrm{j}$ en la red.

$N^{\prime \prime}=$ Número total de nodos en el vecindario del nodo j en la red.

Minimizar $\sum_{k=1}^{K v} \sum_{v=1}^{V} \sum_{j=1}^{N} \sum_{i=1}^{N} D_{i j} X_{i j j k}+D_{i j} Y_{i j v k}$

sujeto a:

$\sum_{k=1}^{K v} \sum_{v=1}^{V} \sum_{(i, j) \in A} X_{i j v k}=1 \quad \forall(i, j) \in A$

$\sum_{k=1}^{K v} \sum_{v=1}^{V} \sum_{(i, j) \in A} X_{i j v k}+\sum_{k=1}^{K v} \sum_{v=1}^{V} \sum_{(j, i) \in A} X_{j i v k}=1 \quad \forall(i, j) \in A \quad(j, i) \in A_{(1 \mathrm{~b})}$

$\sum_{k=1}^{K v} \sum_{v=1}^{V} \sum_{j \in I}^{N^{\prime}} X_{i j j k}+Y_{i j j k} \geq 1 \quad i=1,2, \ldots, N$

$\sum_{k=1}^{K v} \sum_{v=1}^{V} \sum_{i \in I^{\prime}}^{N^{\prime \prime}} X_{i j \jmath k}+Y_{i j j k} \geq 1 \quad j=1,2, \ldots, N$

$\sum_{i=1}^{N} \sum_{j=1}^{N} Q_{i j}\left(\sum_{(i, j) \in A} X_{i j v k}\right) \leq C_{v} \quad v=1,2, \ldots V$

$\sum_{k=1}^{K v} \sum_{j=1}^{N} \sum_{i=1}^{N}\left(t c_{i j}+t d_{i j}\right) X_{i j v k}+\sum_{k=1}^{K v} \sum_{j=1}^{N} \sum_{i=1}^{N} t d_{i j} Y_{i j v k} \leq T_{v k} \quad v=1,2, \ldots V$ 


$$
\begin{aligned}
& \sum_{j \in I}^{N^{\prime}} X_{i j v k}-\sum_{j \in I}^{N^{\prime}} X_{j i v k}+\sum_{j \in I}^{N^{\prime}} Y_{i j \nu k}-\sum_{j \in I}^{N^{\prime}} Y_{j i v k}=0 \quad v=1,2, . . V ; \quad g=1,2, . . K_{V} ; \quad i=1,2, .(N \\
& X_{i j v k} \geq 0 \quad ; \quad Y_{i j v k} \geq 0
\end{aligned}
$$

El objetivo básico de un problema de asignación de rutas de vehículos en un sistema de recolección de residuos sólidos en la acera es obtener un conjunto de rutas de recolección desde un origen a varios puntos de oferta (arcos), minimizando la distancia total recorrida. El primer grupo de restricciones asegura que todos los arcos en la red de recolección (variable Xijvk) van a ser recorridos solamente una vez, de acuerdo a la tipología de la red se presentan dos tipos de restricciones: una para cuando la red es simétrica (1a) y otra para cuando la red es asimétrica (1b). La restricción (2) asegura que cada nodo puede ser visitado por un solo vehículo recolector a la vez y que cada nodo debe ser visitado al menos por un vehículo, además garantiza que el número de vehículos que entran y salen de cada nodo en la red es el mismo. La capacidad de la flota de vehículos esta dada por la restricción (3). La restricción (4) limita el tiempo total de recolección disponible en un día. La restricción de conservación de flujo (5) garantiza que el vehículo que abandona el nodo debe ser el mismo vehículo que ingresa al nodo de tal forma que un nodo solo puede ser visitado por un vehículo en el programa de recolección. La restricción (6) garantiza que todas las variables de decisión son enteras y no negativas. La restricción de rompimiento de subtoures en este tipo de problema no es aplicada, esto se debe a que la restricción de visitas a nodos (2) es mayor o igual a uno lo cual permite que un arco se repita en la solución tantas veces como sea necesario para que el modelo alcance un valor óptimo.

\subsection{Supuestos}

- Todos los arcos en la red de recolección deben ser recorridos una vez.

- Para que se genere un Tour de Euler la red debe ser conectada, para garantizar que el recorrido inicie en el punto más cercano a la base de operaciones ó garaje y finalice en el punto más cercano al sitio de disposición final es necesario agregar un arco ficticio $(\mathrm{t}, \mathrm{s})$ que conecte el último punto de recolección de la microruta con el primero. Este arco se conoce en la teoría de grafos como un arco de tránsito, sus valores de distancia, demanda, tiempo de carga y tiempo de desplazamiento deben tender a cero para que su incidencia sea mínima en el resultado final.
- Solo existe un lugar de disposición final de residuos.

- El trabajo se concentra sólo en el estudio del microruteo por tal razón los tiempos de viaje entre garaje microruta, microruta - relleno y relleno - garaje, así como el tiempo en el relleno sanitario, no serán tenidos en cuenta.

\subsection{Construcción del itinerario}

El itinerario a seguir por cada ruta debe iniciar el recorrido en el punto más cercano al garaje, minimizar los giros en U y a la izquierda, evitar recorrer los arcos en más de una oportunidad y terminar el recorrido en el punto más cercano al sitio de disposición final, según lo dispuesto por las autoridades en MDE-RAS [16]. Para ello se propone el siguiente Algoritmo de Secuenciamiento:

Paso 1. Con base en los resultados del ARV, elabore una lista con todos los arcos disponibles (i, j)

Paso 2. Elimine de la lista de disponibles el $\operatorname{arco}(t, \mathrm{~s})$ que une el nodo final $t$ con el nodo inicia $s$.

Paso 3. Actualice la lista de arcos disponibles (i, j).

Paso 4. Si la lista de arcos disponibles esta vacía vaya a (10) de lo contrario continué en (5)

Paso 5. Ubique los arcos candidatos cuyo nodo origen i sea el último $\mathrm{j}$ asignado.

Paso 6. Seleccione el arco (i,j) que permita ir de frente y vaya a (3), de lo contrario vaya a (7).

Paso 7. Seleccione el arco $(\mathrm{i}, \mathrm{j})$ que permita girar a la derecha y vaya a (3), de lo contrario vaya a (8)

Paso 8. Seleccione el arco $(\mathrm{i}, \mathrm{j})$ que permita girar a la izquierda y vaya a (3), de lo contrario vaya a (9)

Paso 9. Seleccione el arco $(\mathrm{i}, \mathrm{j})$ que permite girar en $\mathrm{U}$ asígnelo y vaya a (3).

Paso 10. Elabore el itinerario de recolección final con base en el orden dado a cada arco. 


\section{Resultados}

\subsection{Validación}

El problema de asignación de rutas de vehículos tiene dos estrategias de solución: una permite la formación de un gran tour que recorra toda la zona y la otra permite que se generen rutas que interactúen entre sí. El modelo fué implementado bajo distintos escenarios en los cuales se modifican las restricciones de tiempo y capacidad con el fin de analizar su comportamiento y determinar las variaciones entre los recorridos generados en cada microruta.

Al utilizar la estrategia de formar un gran tour con un solo vehículo y restricciones de capacidad y tiempo ilimitadas (Escenario 1) se generan recorridos continuos, óptimos desde el punto de vista matemático, fáciles de implementar y controlar a nivel administrativo.

Cuando se utiliza la estrategia de formación de rutas que interactúan entre sí se pueden presentar dos situaciones:

1. Sí las restricciones de tiempo y capacidad tienen suficiente holgura (Escenario 2), se generan rutas continuas, óptimas desde el punto de vista matemático y relativamente fáciles de supervisar, aunque para su implementación se deban tomar decisiones de carácter administrativo en cuanto al punto de inicio y finalización de alguno de los recorridos.

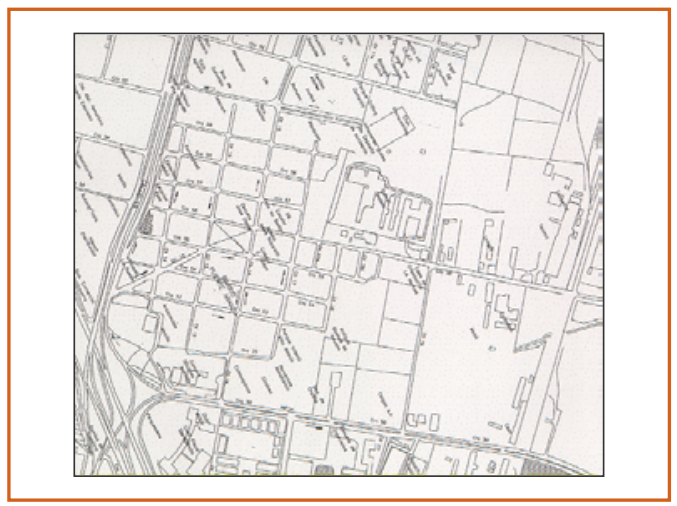

Figura 2. Zona de Estudio - Puente Aranda, Bogotá
2. Sí las restricciones de tiempo y capacidad están muy restringidas o con poca holgura (Escenario 3), dan origen a la formación de rutas continuas y discontinuas, óptimas desde el punto de vista matemático y con alguna dificultad al momento de ser implementadas ya que es necesario definir el punto de inicio y finalización de alguno de los recorridos, así como de los tramos que deben ser agregados para garantizar la continuidad del itinerario.

\subsection{Aplicación}

El modelo ARV fue implementado en una zona de recolección de Santafé de Bogotá (figura 2) con el ánimo de obtener resultados que permitan realizar comparaciones con respecto a dos soluciones conocidas, una ruta generada por un Sistemas de Información Geográfica y la ruta utilizada actualmente por la empresa prestadora del servicio de recolección (Rotf [17]).

La ruta generada por el Modelo ARV (Figura 3) presenta los siguientes resultados (Tabla 1). En términos de distancia genera un ahorro de $458.13 \mathrm{mts}$ respecto a la ruta actual. En términos de tiempo se logra un ahorro de 4.47 minutos el cual podría incrementarse notoriamente en caso de llegar a utilizar factores de penalización por giros a la izquierda, en $\mathrm{U}$ y arcos repetidos. En cuanto a la estructura del itinerario, se obtiene un ahorro del $50 \%$ en lo que se refiere a los giros en $\mathrm{U}$ y a la izquierda, los cruces de calle (continuar de frente) aumentan lo cual

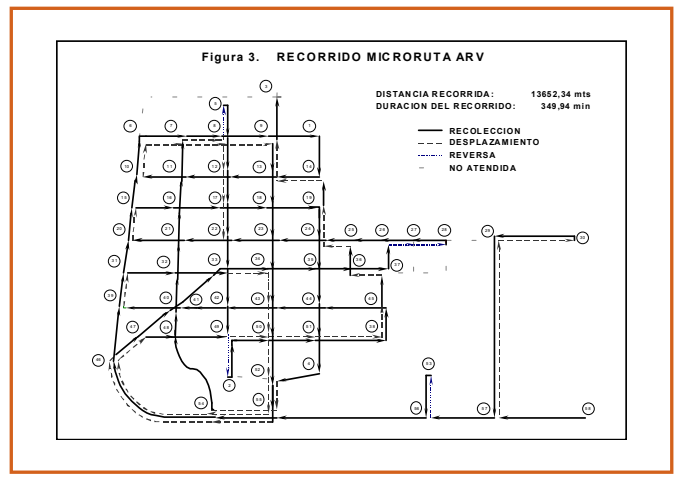

Figura 3. Recorrido Microruta ARV 


\begin{tabular}{|c|c|c|c|c|}
\hline \multirow{6}{*}{$\begin{array}{l}\text { DISTANCIA } \\
\text { (metros) }\end{array}$} & \multirow[t]{2}{*}{ PARÁMETRO } & \multicolumn{3}{|c|}{ MICRORUTA } \\
\hline & & EMPRESA & SIG & ARV \\
\hline & Recorrido recolección & 9326,93 & 9365,61 & 9365,61 \\
\hline & Recorrido desplazamiento & 4783,54 & 4583,55 & 4286,73 \\
\hline & Distancia total recorrida & 14110,47 & 13949,16 & 13652,34 \\
\hline & Ahorro en metros & & 161,31 & 458,13 \\
\hline \multirow{4}{*}{$\begin{array}{l}\text { TIEMPO } \\
\text { (minutos) }\end{array}$} & Red de recolección & 321,60 & 321,86 & 321,86 \\
\hline & Red de desplazamiento & 32,81 & 30,19 & 28,08 \\
\hline & Tiempo total del recorrido & 354,41 & 352,05 & 349,94 \\
\hline & Ahorro en minutos & & 2,36 & 4,47 \\
\hline \multirow{7}{*}{$\begin{array}{c}\text { ESTRUCTURA } \\
\text { DEL ITINERARIO } \\
\text { (número de veces) }\end{array}$} & Cruce de calle - Frente & 69 & 51 & 79 \\
\hline & Giro a la derecha & 40 & 52 & 33 \\
\hline & Giro a la izquierda & 19 & 23 & 10 \\
\hline & Giro en U - Reversa & 10 & 5 & 5 \\
\hline & Total arcos recorridos & 138 & 131 & 127 \\
\hline & Arcos de recolección & 89 & 90 & 90 \\
\hline & Arcos de tránsito (repetidos) & 49 & 41 & 37 \\
\hline \multirow{6}{*}{$\begin{array}{l}\text { PENALIZACIONES } \\
\text { (minutos) }\end{array}$} & Giro a la izquierda (10 seg) & 3,167 & 3,833 & 1,667 \\
\hline & Giro en U - Reversa (30 seg) & 7,500 & 3,750 & 3,750 \\
\hline & Arcos repetidos $(5 \mathrm{seg})$ & 4,083 & 3,417 & 3,083 \\
\hline & Total penalizaciones (min) & 14,750 & 11,000 & 8,500 \\
\hline & Tiempo total (min) & 369,160 & 363,050 & 358,440 \\
\hline & Ahorro en minutos & & 6,110 & 10,720 \\
\hline
\end{tabular}

quiere decir que se incrementan los trayectos en línea recta y el número de arcos repetidos (deadheading) se reduce en un $25 \%$. Vale la pena resaltar que aunque se logran mejorar los resultados estos no son altamente significativos debido a que se están comparando contra buenas soluciones. Sin embargo tiene grandes beneficios potenciales al ser aplicado a gran escala o en áreas poco analizadas, pues se puede lograr una reducción sustancial de costos teniendo en cuenta el tamaño de la zona y el volumen de residuos a ser recolectados.

\subsection{Generación de itinerarios}

El Modelo ARV también puede emplearse en macroruteo. Específicamente en lo relacionado con la generación de distritos de recolección siguiendo para ello la metodología planteada por el algoritmo Male \& Liebman [5], pues basta con implementar el modelo ARV como parte del algoritmo en su primera etapa obteniendo un grafo par y luego continuando con la metodología descrita a continuación:

Paso 1. Convertir la red en un grafo de grado par, implementando el modelo ARV.
Paso 2. Generar ciclos lo más pequeños posibles.

Paso 3. Convertir la red de arcos en una red con los ciclos asignados.

Paso 4. Construir el mínimo árbol cobertor capacitado.

Paso 5. Definir las rutas en la red original.

\section{Conclusiones}

El modelo de asignación de rutas de vehículos para un sistema de recolección de residuos sólidos en la acera que se ha presentado, ofrece la posibilidad de trabajar con dos conjuntos de variables de decisión enteras las cuales facilitan efectuar cambios en la función objetivo y en las restricciones. También permiten identificar claramente la actividad que realiza la tripulación en su respectivo vehículo. Esta diferenciación facilita la elaboración y trazado del itinerario de recolección, generando resultados por separado para cada vehículo respecto a la distancia recorrida en cada red y su permanencia en la misma, a la vez que permite determinar la tasa de utilización por vehículo y el tiempo empleado por tripulación.

El modelo ARV genera una mejor ruta que la sugerida 
por el SIG y la empleada actualmente por la empresa prestadora del servicio de recolección, obteniéndose una ruta de menor longitud que se recorre en menos tiempo. Además al emplear el algoritmo de secuenciamento se minimizan considerablemente los giros prohibidos.

Se observa que la estrategia consistente en la formación de un gran tour es la más indicada y aconsejable para el trazado de rutas de recolección ya que provee una solución óptima que facilita su implementación y control a nivel administrativo.

Esta formulación también puede ser aplicada al problema de asignación de rutas sobre nodos eliminando las restricciones de cobertura y cambiando el sentido de las restricciones de visitas a nodos.

Al usar modelo ARV propuesto con el algoritmo Male\&Liebman se pueden generar distritos o zonas de recolección para cualquier tipo de red.

\section{Futuras Líneas de Investigación}

Como alternativas para posteriores estudios se plantea: La posibilidad de integrar el modelo ARV como parte de un SIG, agregar nuevas variables de decisión al problema que permitan contar con más de un sitio de disposición final y varias estaciones de transferencia. También se puede diseñar una herramienta computacional que permita implementar el algoritmo de secuenciamiento e involucrar factores de penalización por giros en U, giros a la izquierda ó desplazamientos en reversa. Finalmente se podría considerar la posibilidad de utilizar el modelo en problemas afines tales como el de la repartición y/o entrega de periódicos o correo, barrido manual y mecánico de calles y barrido de nieve en temporadas invernales.

\section{BiblografíA}

1 TSCHOBANOGLOUS G., H. THEISEN, y S. VIGEL. Integrated Solid Waste Management. Mc Graw-Hill, 1993.

2 BELTRAMI E. \& L. BODIN. Networks and Vehicle Routing for Municipal Wasre Collection. Networks, 4, Nov, 1974, p 65 - 94.

3 SHUSTER K., D. SCHUR. Heuristic Routing for Solid Waste Collection Vehicles, Publication SW-113, U.S. Enviromental Protection Agency, Washington, 1974.

4 MALE James, Jon LIEBMAN. Districting and Routing for Solid Waste Collection. Journal of the Enviromental
Engineering División. Vol 164, Febrero 1978.

5 BODIN L., B. GOLDEN. Classification in Vehicle Routing and Scheduling, Networks, Vol 11, John Willey \& Sons, 1981.

6 EISENTEIN Donald, Ananth IYER. Garbage Collection in Chicago: A Dinamic Scheduling Model.Mangemnet Science, Vol 43, No 7, Julio 1997.

7 OR Ilham. y Kriton CURI. "Improving the Efficiency of the Solid Waste Collection System in Izmir, Turkey, Throught Mathematical Programming. Waste Management \& Research, 11, 1993, p 297-311.

8 MASSIE K. Using GIS to Improve Solid Waste Mangement and Recyclig Programs, Procedures, 1995 ESRI User Conf., 18.

9 CARGIN J. \& J. DAWYER. Pennsylvania`s low-level Radioactive Waste Disposal Facility Siting Project: Special GIS Operations, Procedures, 1995 ESRI User Conf., 162

10 OSEGUEDA R, GARCIA A Y S ASHUR. GIS-Based Network Routing Procedures for Overweight and Oversized Vehicles, Journal of Transportation Engineering, July-August, 1999, p 324 - 331

11 CHANG N.B., LU H.Y., \& L.Y. WEI. GIS Technology for Vehicle Routing and Scheduling in Solid Waste Colection Systems, Journal of Environmental Engineering, 123, 9, September, 1997, p 901 - 910.

12 LAPORTE G. The Traveling Salesman Problem: An overview of exact and approximate algorithms. European Journal of Operational Research, 59, 1992, p 231 247.

13 EDMONS J. \& E.L. JOHNSON, "Matching Euler and the Chinese Postman" Mathematical Programming, 5, 1973, p 88-124.

14 EISELT H, GENDREAU M y LAPORTE G. Arc Routing Problems, part I: The chinese postman problem. Operations Research. 43, 1995, p 231 - 242.

15 EVANS James y Edward MINIEKA. Optimization Algorithms for Networks and Graphs, Marcel Dekker Inc, New York, 1992, p 278-315.

16 MINISTERIO DE DESARROLLO ECONÓMICO. Reglamento Técnico del Sector de Agua Potable y Saneamiento Básico. 1998.

17 ROTH Hans. Optimización de Rutas de Recolección de Basuras Utilizando Sistemas de Información Geográfica, Ingeniería Civil, Universidad de los Andes, 1999. 